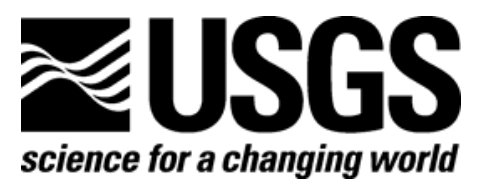

\title{
Log ASCII Standard (LAS) files for geophysical wireline well logs and their application to geologic cross sections through the central Appalachian basin
}

By Robert D. Crangle, Jr.

Open File Report 2007-1142 


\section{U.S. Department of the Interior DIRK KEMPTHORNE, Secretary}

\section{U.S. Geological Survey \\ Mark D. Myers, Director}

\section{U.S. Geological Survey, Reston, Virginia 2007}

For product and ordering information:

World Wide Web: http://www.usgs.gov/pubprod

Telephone: 1-888-ASK-USGS

For more information on the USGS - the Federal source for science about the Earth, its natural and living resources, natural hazards, and the environment:

World Wide Web: http://www.usgs.gov

Telephone: 1-888-ASK-USGS

Suggested citation:

Crangle, R.D., Jr., 2007, Log ASCII Standard (LAS) files for geophysical wire line well logs and their application to geologic cross sections through the central Appalachian basin: United States Geological Survey Open File Report 2007-1142, 14 p.

Any use of trade, product, or firm names is for descriptive purposes only and does not imply endorsement by the U.S. Government. Use of trade names in this report is for descriptive purposes only and does not imply endorsement by the USGS.

Although this report is in the public domain, permission must be secured from the individual copyright owners to reproduce any copyrighted material contained within this report. 


\section{Contents}

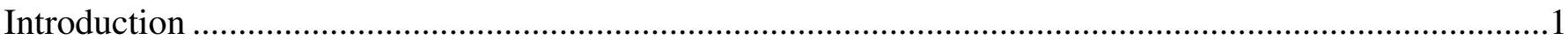

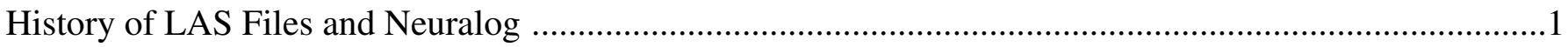

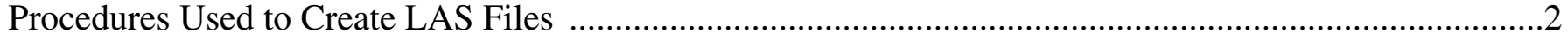

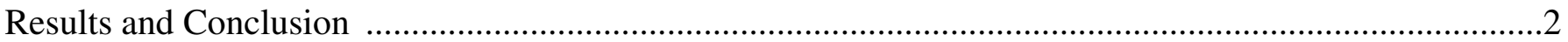

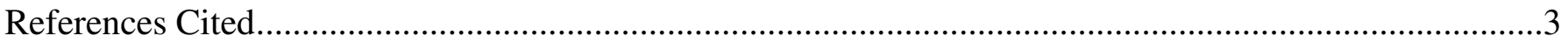

\section{Figures}

1. Location map for regional cross-sections $D-D^{\prime}$ and $E$ - $E$ ' (red lines). Well locations represented as black dots, numbers correspond to well data listed in Tables 1 and 2 ....................................................

2. Example of an LAS file of gamma ray data for the \#1 Windbigler well, Morrow County, OH. Selected data from a depth range of 120.5 to 140.5 feet …………...........................................................

3. Selected segment of the Windbigler \#1 gamma ray log used to define lithology and correlate stratigraphy. The image file (left) was imported into Neuralog where it was traced, digitized (right), and converted to an LAS file. The resulting LAS file (Fig. 2) was imported into GeoGraphix Prizm, where it was converted to a digital image, exported as a WMF image file, then imported into Adobe Illustrator and included as part of the geologic cross section $E-E^{\prime}$. The resolution for this figure is compromised due to the original condition of the paper well log and the accompanying, captured Neuralog screen-capture (Fig. 4)

4. Selected segment of geologic cross section $E-E$ ' showing the location of the No. 1 Windbigler well and a digitized gamma ray log of the well (yellow). The area in blue corresponds to the data shown in figures 2 and 3 .............................................................................................................

\section{Tables}

1. Sixteen Log ASCII Standard (LAS) files for geologic cross section E-E' through the Appalachian basin from Wood County, Ohio, to Pendleton County, West Virginia ........................................................8

2. Log ASCII Standard (LAS) files for geologic cross section D-D' through the Appalachian basin from Sandusky County, Ohio, to Hardy County, West Virginia 


\title{
Log ASCII Standard (LAS) files for geophysical wire line well logs and their application to geologic cross sections through the central Appalachian basin
}

\author{
By Robert D. Crangle, Jr. ${ }^{1}$
}

\section{Introduction}

The U.S. Geological Survey (USGS) uses geophysical wireline well logs for a variety of purposes, including stratigraphic correlation (Hettinger, 2001, Ryder, 2002), petroleum reservoir analyses (Nelson and Bird, 2005), aquifer studies (Balch, 1988), and synthetic seismic profiles (Kulander and Ryder, 2005). Commonly, well logs are easier to visualize, manipulate, and interpret when available in a digital format.

In recent geologic cross sections $E-E^{\prime}$ and $D-D^{\prime}$, constructed through the central Appalachian basin (Ryder, Swezey, and others, in press; Ryder, Crangle, and others, in press), gamma ray well log traces and lithologic logs were used to correlate key stratigraphic intervals (Fig. 1). The stratigraphy and structure of the cross sections are illustrated through the use of graphical software applications (e.g., Adobe Illustrator). The gamma ray traces were digitized in Neuralog (proprietary software) from paper well logs and converted to a Log ASCII Standard (LAS) format. Once converted, the LAS files were transformed to images through an LAS-reader application (e.g., GeoGraphix Prizm) and then overlain in positions adjacent to well locations, used for stratigraphic control, on each cross section.

This report summarizes the procedures used to convert paper logs to a digital LAS format using a third-party software application, Neuralog. Included in this report are LAS files for sixteen wells used in geologic cross section $E-E^{\prime}$ (Table 1) and thirteen wells used in geologic cross section $D-D^{\prime}$ (Table 2).

\section{History of LAS files and Neuralog}

Prior to the introduction of the LAS format, geophysical well-log data were recorded on magnetic tape and reviewed in a non-standardized binary format. In 1990, the Canadian Well Logging Society designed the LAS ASCII-type system for local Canadian markets to standardize the binary format used to digitize well logs. The simplicity and flexibility of the LAS ASCII-type encoding quickly led to its worldwide acceptance and use (personal communication, Kenneth Heslop, Canadian Well Logging Society, 10/17/2005). As shown in figure 2, the LAS format often begins with a header, followed by columns of values that

${ }^{1}$ U.S. Geological Survey, Reston, Virginia 20192 
describe specific measurements (e.g., gamma ray log in API radiation units) at given depths (Canadian Well Logging Society, 1990, http://cwls.org/docs/LAS12_Standards.txt).

The Neuralog software program (Neuralog, Inc., 1992), is an autovectorizing application used to create LAS files. The application is capable of generating an LAS file from a scanned well-log, imported as an image file (e.g., Tagged Image File Format) and digitized through a combination of an autovectorizing algorithm and user-defined manual edits. Once vectorized, Neuralog converts the digital image to an LAS file, which can then be used in a variety of other LAS-reader applications (e.g., GeoGraphix Prizm, Microsoft Excel, GeoTools QuickSyn, etc.). The conversion of a paper well log to a digital LAS file through Neuralog is quite useful, particularly for older logs where original digital data is limited or nonexistent.

\section{Procedures used to create LAS files}

Gamma ray logs, used to construct geologic cross sections $E-E^{\prime}$ and $D-D^{\prime}$ (Fig. 1), were converted to LAS-format files through Neuralog. The LAS files, included in Tables 1 and 2, were created from scanned Tagged Image File Format (TIFF) files (Fig. 3) that were in turn converted to an LAS format (text file) via Neuralog. Once transformed to an LAS format, the file was accessible in an LAS viewer (e.g., GeoGraphix Prizm) where it was exported as a Windows Metafile format (WMF) image file. A graphical illustration application (e.g., Adobe Illustrator), was used to import the WMF file for further editing, which included scaling and placement (Fig. 4). Multiple quality-check verifications were made throughout the process to insure consistency between the original paper logs and the newly created digital logs.

Prior to the development of Neuralog, non-digital geophysical well logs were scanned, saved as TIFF files, manually traced in a graphical illustration application and placed within an appropriate stratigraphic cross-section illustration (e.g., Hettinger, 2001, Ryder 2002). This process produced vectorized, editable images, but no usable coordinates (e.g., X,Y data). With Neuralog, a more accurate and fully editable image, coupled with a trans-application digital data file, is achieved. The Neuralog vectorization process offers a significant savings in time and the resultant LAS data file may be used in a variety of other applications.

\section{Results and Conclusion}

The autovectorization and LAS conversion of paper geophysical wireline well logs provides significant assistance in the correlation, creation, presentation, and digitization of geologic cross sections (Fig. 4). The integration of Neuralog, an LAS-file reader, and a graphical illustration application offers an effective and accurate process whereby non-digital paper well logs are converted to digital LAS files. These same procedures will be used to produce digital images of geophysical well logs for additional cross sections in the Appalachian basin and other localities. 


\section{References Cited}

Balch, A.H., ed., 1988, A seismic-stratigraphic investigation of the Madison and associated aquifer - Application to ground-water exploration, Powder River Basin, MontanaWyoming: U.S. Geological Survey Professional Paper 1330, 99p., 1 plate.

Canadian Well Logging Society, 1990. http://cwls.org/docs/LAS12_Standards.txt.

Hettinger, R.D., 2001, Subsurface correlations and sequence stratigraphic interpretations of Lower Silurian strata in the Appalachian basin of northeast Ohio, southwest New York, and northwest Pennsylvania: U.S. Geological Survey Geologic Investigations Series Map I-2741, 1 sheet, scale about 1:2,000,000, 22-p. text.

Kulander, C.S. and Ryder, R.T., 2005, Regional seismic lines across the Rome trough and Allegheny Plateau of northern West Virginia, western Maryland, and southwestern Pennsylvania: U.S. Geological Survey Geologic Investigations Series Map I-2791, 2 sheets, 1 pamphlet, 9p.

Nelson, P.H. and Bird, K.J., 2005, Porosity-depth trends and regional uplift calculated from sonic logs, National Petroleum Reserve in Alaska: U.S. Geological Survey Scientific Investigations Report 2005-5051, 28p.

Ryder, R.T., 2002, U.S. Geological Survey Miscellaneous Investigations Series Map I-2200, Stratigraphic framework of Cambrian and Ordovician rocks in the central Appalachian Basin from Lake County, Ohio, to Juniata County, Pennsylvania, Online Version 1.0, U.S. Geological Survey, Reston, VA, http://pubs.usgs.gov/i-maps/i-2200/.

Ryder, R.T., Crangle, Jr., R.D., Trippi, M.H., Swezey, C.S., Lentz, E.E., Rowan, E.L., and Hope, R.S., in press, Geologic cross section D-D' through the Appalachian basin from the Findlay arch, Sandusky County, Ohio to the Valley and Ridge province, Hardy County, West Virginia: U.S. Geological Survey Scientific Investigative Series Map I-

Ryder, R.T., Swezey, C.S., Crangle, R.D., Jr., and Trippi, M.H., in press, Geologic cross section E-E' through the Appalachian basin from the Findlay arch, Wood County, Ohio, to the Allegheny structural front, Pendleton County, West Virginia, U.S. Geological Survey Scientific Investigations Series Map. 


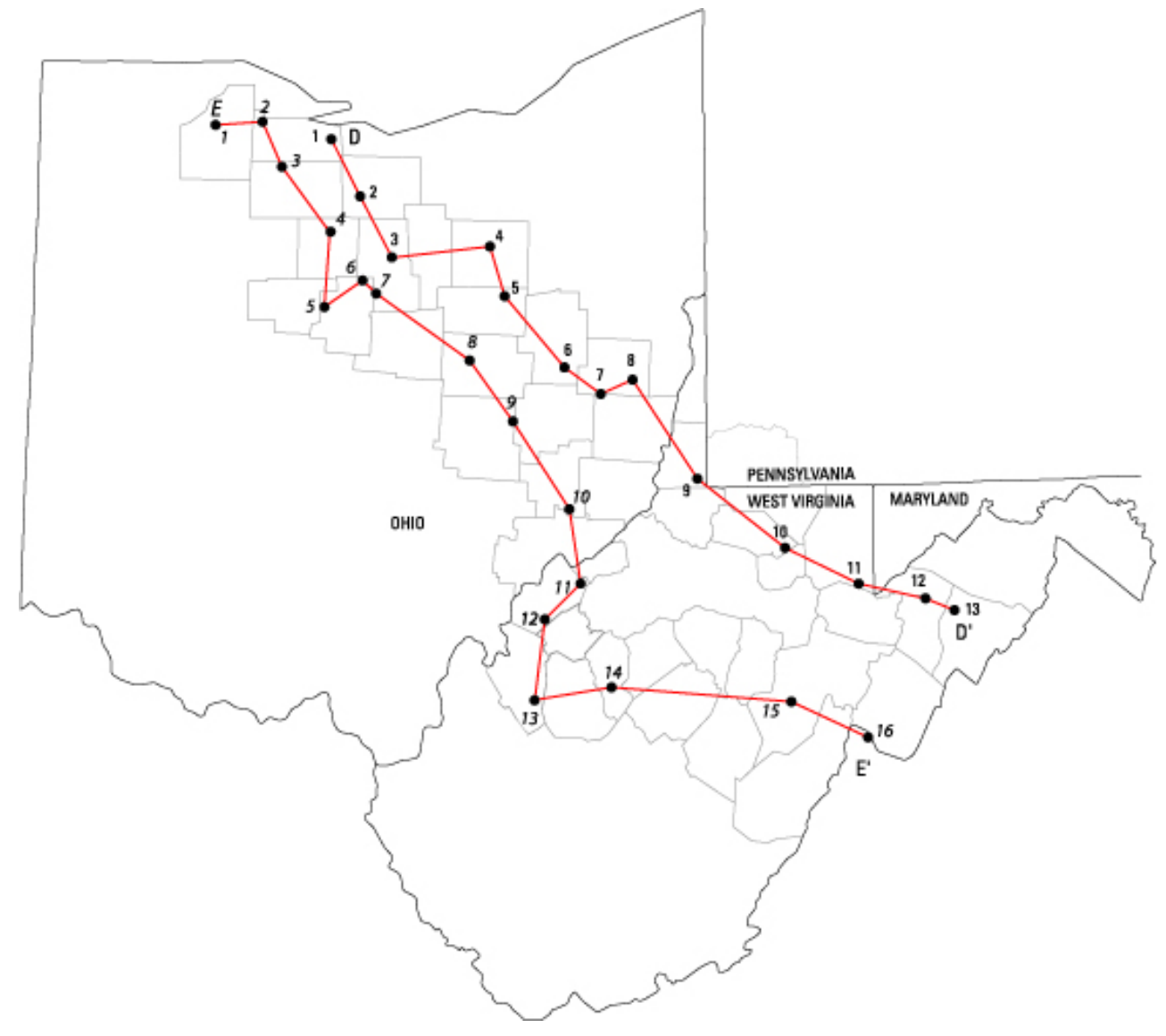

Figure 1. Location map for regional cross-sections $D-D^{\prime}$ and $E-E$ ' (red lines). Well locations represented as black dots, numbers correspond to well data listed in Tables 1 and 2. 


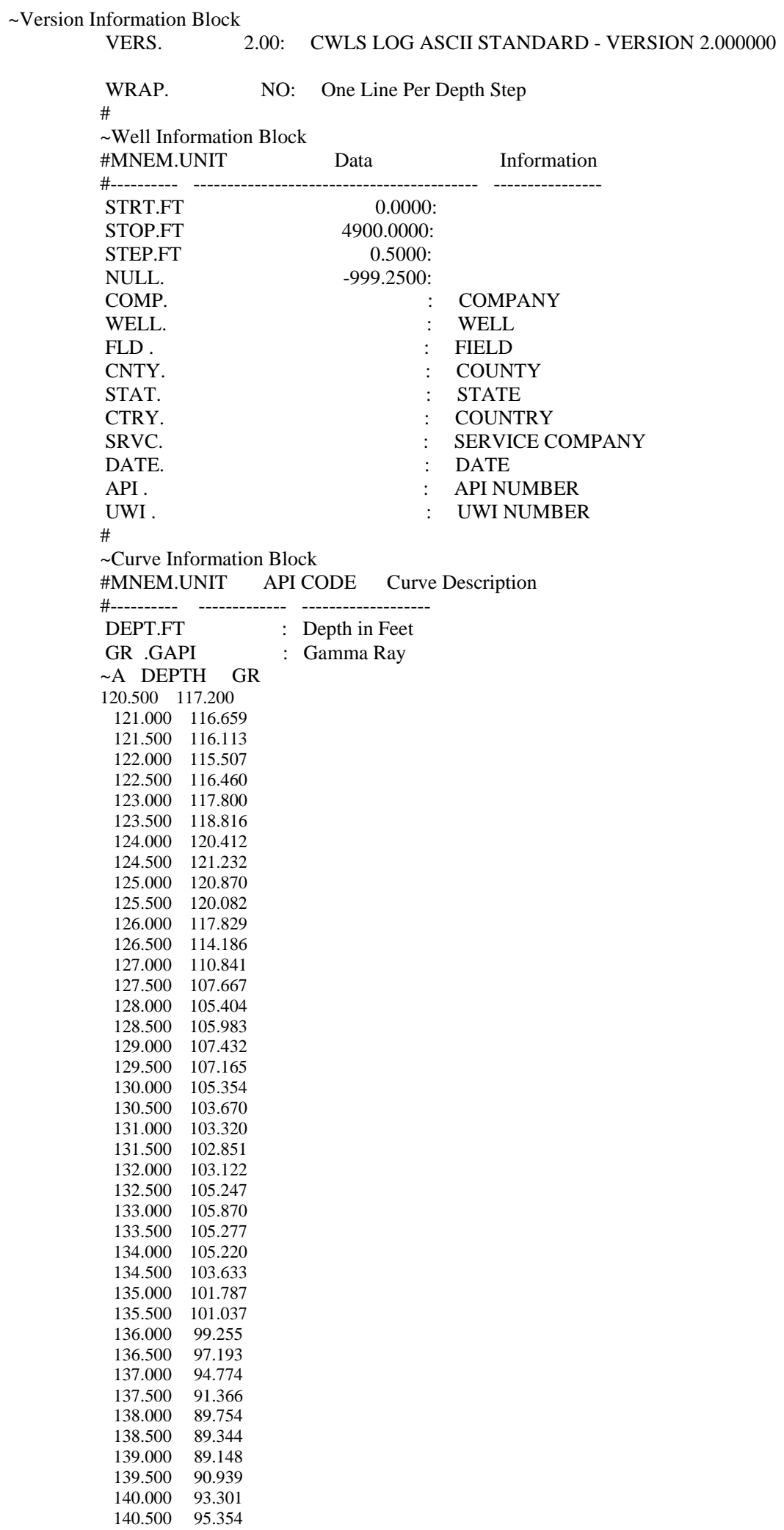

Figure 2. Example of an LAS file of gamma ray data for the \#1 Windbigler well, Morrow County, OH. Selected data from a depth range of 120.5 to 140.5 feet. 


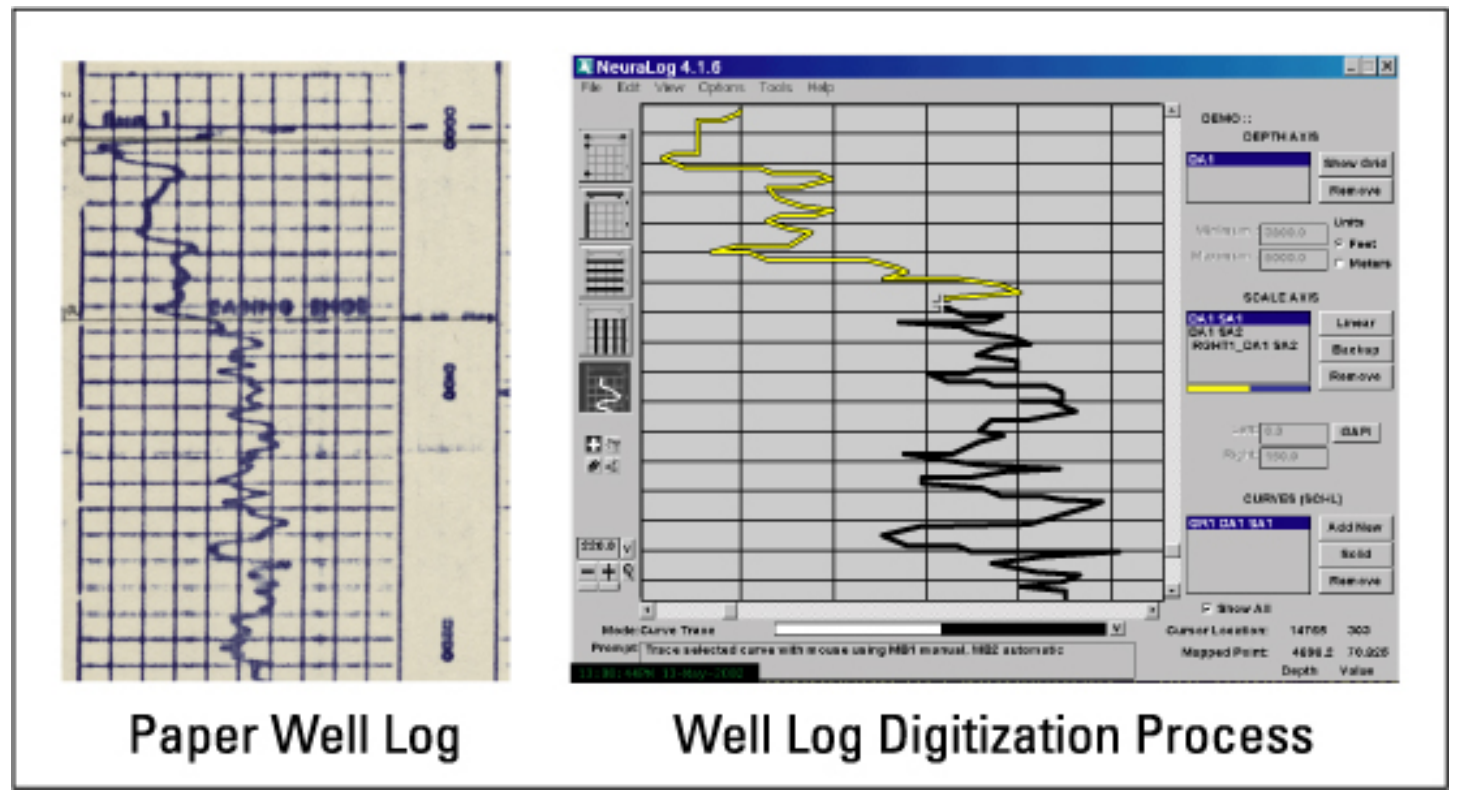

Figure 3. Selected segment of the Windbigler \#1 gamma ray log used to define lithology and correlate stratigraphy. The image file (left) was imported into Neuralog where it was traced, digitized (right), and converted to an LAS file. The resulting LAS file (Fig. 2) was imported into GeoGraphix Prizm, where it was converted to a digital image, exported as a WMF image file, then imported into Adobe Illustrator and included as part of the geologic cross section $E$ $E^{\prime}$. The resolution for this figure is compromised due to the original condition of the paper well $\log$ and the original native resolution of the Neuralog screen-capture. 


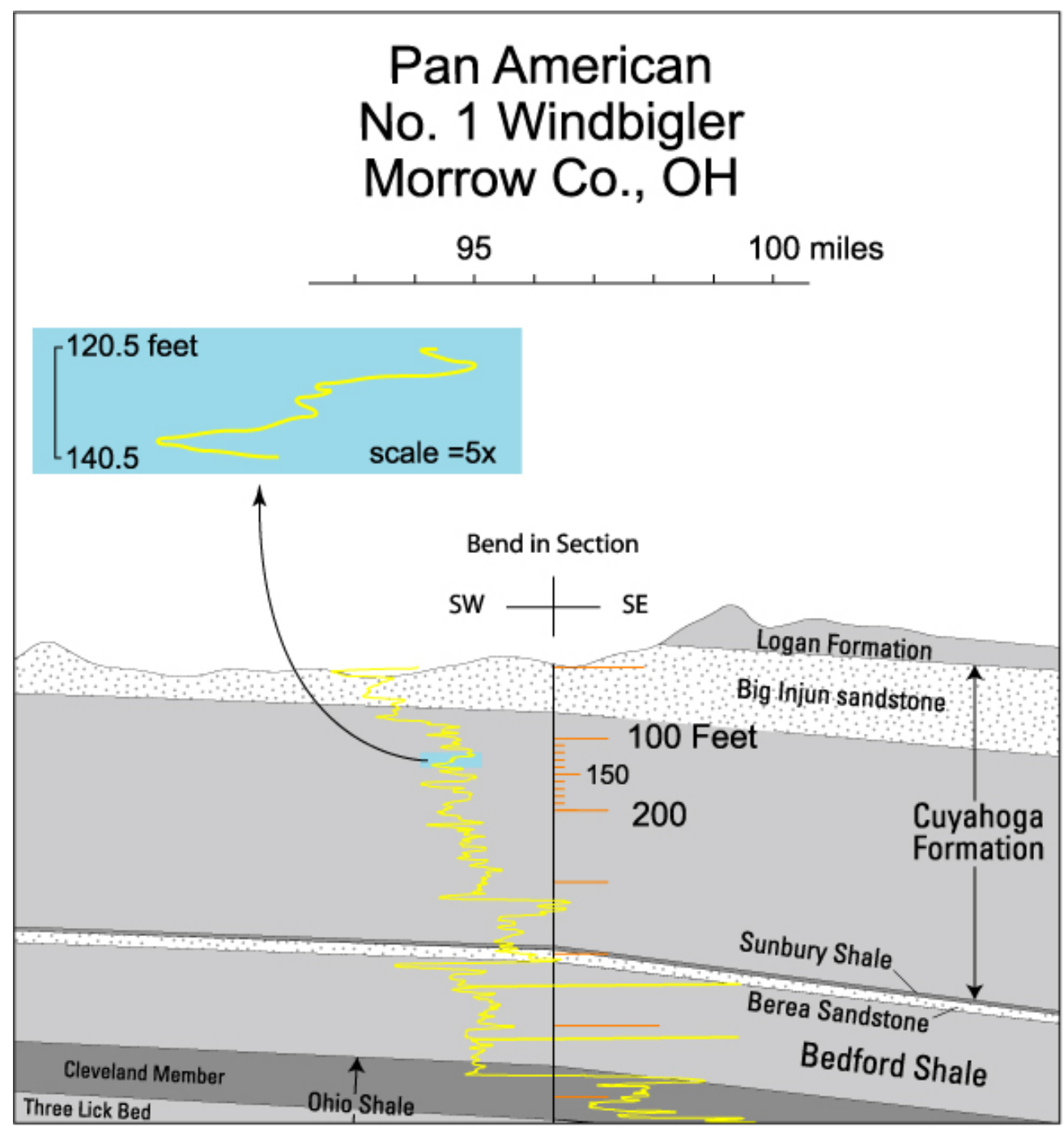

Figure 4. Selected segment of geologic cross section E-E' showing the location of the No. 1 Windbigler well and a digitized gamma ray log of the well (yellow). The area in blue corresponds to the data shown in figures 2 and 3 . 
Table 1. Sixteen Log ASCII Standard (LAS) files for geologic cross section E-E' through the Appalachian basin from Wood County, Ohio, to Pendleton County, West Virginia

\begin{tabular}{|c|c|c|c|c|c|c|c|c|c|}
\hline Well \# & File Name & Well Name & Location & API No. & Latitude & Longitude & TD (ft) & Date(s) of log run(s) & LAS Interval (ft) \\
\hline & A_KM_ark_oil & \begin{tabular}{|l} 
Kin-Ark Oil Co. No. 1 Carter \\
\end{tabular} & Center Township, Wood County, $\mathrm{OH}$, Dunbridge, $\mathrm{OH} 7.5$ ' quadrangle & $34-173-20237$ & 41.415 & -83.609 & 2,821 & $\frac{1 / 111 / 1965 \text { (Run 1, GR-N) }}{4}$ & $0-2,800$ \\
\hline & $\begin{array}{l}\text { A_Kerbel_1 (LAS file from Ohio } \\
\text { Geological Survey, } \\
\text { http://www/ohiodnr.com/geosurvey/ogci } \\
\text { m/petrol/dlg.htm) }\end{array}$ & $\begin{array}{l}\text { i Russell Maguire No.1 Paul } \\
\text { Kerbel }\end{array}$ & $\begin{array}{l}\text { Woodville Township, Sandusky County, } \mathrm{OH}, \\
\text { Elmore, } \mathrm{OH} 7.5^{\prime} \text { quadrangle }\end{array}$ & 34-143-20147 & 41.438 & -83.317 & 2,785 & 11/29/1965 (Run 1, GR-N) & $100.5-2,779.5$ \\
\hline & A_M_and_B_Asphalt & $\begin{array}{l}\text { Ohio Dept. of Nat. Resources } \\
\text { M and B Asphalt Co. }\end{array}$ & $\begin{array}{l}\text { Liberty Township, Seneca County, } \mathrm{OH}, \\
\text { Tiffin North, } \mathrm{OH} 7.5^{\prime} \text { quadrangle }\end{array}$ & $34-147-60840$ & 41.226 & -83.199 & 2,870 & 1985 (Run 1, GR-N) & \\
\hline & $\begin{array}{l}\text { A_Leonhardt (LAS file from Ohio } \\
\text { Geological Survey, } \\
\text { http://wwww.ohiodnr.com/geosurvey/ogci } \\
\text { m/petrol/dlg.htm) }\end{array}$ & $\begin{array}{l}\text { i } \begin{array}{l}\text { HL Hawkins \& HL Hawkins, Jr. } \\
\text { No.1 VE Leonhardt }\end{array} \\
\text {. }\end{array}$ & $\begin{array}{l}\text { Chattield Township, Crawford County, } \mathrm{OH} \text {, } \\
\text { Chatfield, OH } 7.5 \text { ' quadrangle }\end{array}$ & $34-033-20050$ & 40.910 & -82.883 & $3,77$. & $10 / 11 / 1965$ (Run 1, GR-N) & \\
\hline & A_Myers_A & $\begin{array}{l}\text { United Producing Co No.3 } \\
\text { Orie Myers (O \& E Myers) }\end{array}$ & $\begin{array}{l}\text { Canaan Township, Morrow County, } \mathrm{OH}, \\
\text { Denmark, } \mathrm{OH} 7.5 \text { quadrangle }\end{array}$ & 34-117-20012 & 40.571 & -82.920 & 4,100 & $7 / 16 / 1965$ (Run 1) & \\
\hline & A Myers B & $\begin{array}{l}\text { United Producing Co No.3 } \\
\text { orie Myers (O \& E Myers) }\end{array}$ & $\begin{array}{l}\text { Canaan Township, Morrow County, } \mathrm{OH}, \\
\text { Denmark, } \mathrm{OH} \text {.5' quadrangle }\end{array}$ & $34-117-20012$ & 40.571 & -82.920 & 4,100 & $7 / 26 / 1965$ (Run 2) & 2,900-4, \\
\hline & A_Windbigler & $\begin{array}{l}\text { Pan American Petroleum Corp. } \\
\text { No.1 AC Windbigler }\end{array}$ & $\begin{array}{l}\text { Troy Township, Morrow County, } \mathrm{OH}, \\
\text { Blooming Grove, } \mathrm{OH} 7.5^{\prime} \text { quadrangle }\end{array}$ & 34-117-20047 & 40.690 & -82.682 & 4,890 & 10/13/1962 (Run 1) 11/3/1962 (Run 2) & \\
\hline & A_Palmer & Pan American No.1 J Palmer & $\begin{array}{l}\text { Troy Township, Richland County, OH, } \\
\text { Mansfield South, } \mathrm{OH} 7.5 \text { quadrangle }\end{array}$ & 34-139-20289 & 40.648 & -82.590 & 4,775 & 2/28/1964 (Run 1, GR-N), 3/18/1964 (Run 2, GR-N) & \\
\hline & A Lee & Bob Tatum No.1 Edwin L Lee & $\begin{array}{l}\text { Jemferson Township, Coshocoton County, } \mathrm{OH} \text {, } \\
\text { Warsaw, OH 7.5' quadrangle }\end{array}$ & $34-031-22053$ & 40.323 & -82.001 & 6.970 & |6/16/1971 (Run 1, FDC-GR) & $100-6.970$ \\
\hline & A_Marshall & $\begin{array}{l}\text { Lakeshore Pipeline No.1 WR } \\
\text { Marshall }\end{array}$ & $\begin{array}{l}\text { Adams Township, Guersey County, } \mathrm{OH}, \\
\text { Bloomfield, } \mathrm{OH} 7.5^{\circ} \text { quadrangle }\end{array}$ & $34-059-20782$ & 40.037 & -81.720 & 8,602 & 4/177-18/1961 (Run 1, L-GR-N), 5/31/1961 (Run 2, L-GR-N) & $100-8$ \\
\hline & $\begin{array}{l}\text { A_Ullman (LAS file from Ohio } \\
\text { Geological Survey, } \\
\text { http://wwww.ohiodnr.com/geosurvey/ogci } \\
\text { m/petrol/dlg.htm) }\end{array}$ & Amerada No.1 Robert Ullman & Elk Township, Noble County, OH, Dalzell, OH 7.5' quadrangle & 34-121-21278 & 39.611 & -81.347 & 11,44 & $\begin{array}{l}8 / 16 / 1966 \text { (Run 1, GR-N), } \\
(\text { Run 3, GR-N) }\end{array}$ & -11, \\
\hline & A_Hope_Power & $\begin{array}{l}\text { Hope Natural Gas No.9634 } \\
\text { Power Oil Co. }\end{array}$ & Walker District, Wood County, WV, Willow Island, WV 7.5' quadrangle & $47-107-00351$ & 39.255 & -81.272 & 13,327 & 1/13-14/1955 (Run 3-GR) & $100-12,300$ \\
\hline 12 & A_Deem & $\begin{array}{l}\text { Exxon Corporation No.1 } \\
\text { Howard H Deem Et Ux }\end{array}$ & Steele District, Wood County, WV, Rockport, WV 7.5' quadrangle & 47-107-00756 & 39.081 & -81.508 & 13,266 & $\begin{array}{l}10 / 13 / 1976 \text { (Run 1,IGR), } 10 / 281 / 1976 \text { (Run 2, IGR), 11/22/1976 } \\
\text { (Run 3, IGR), 11/29/1976 (Run 4, IGR) }\end{array}$ & $100-13,200$ \\
\hline$1 2 \longdiv { 2 }$ & A Deem B & $\begin{array}{l}\text { Exxon Corporation No.1 } \\
\text { Howard H Deem Et Ux }\end{array}$ & $\begin{array}{l}\text { Steele District, Wood County, WW, } \\
\text { Rockport, WV 7.5' quadrangle }\end{array}$ & $47-107-00756$ & 39.081 & -81.508 & 13,266 & 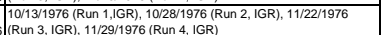 & $8.800-13,240$ \\
\hline & A McCoy & $\begin{array}{l}\text { Exxon Corporation No. } 1 \text { Walter } \\
\text { McCoy Et Al }\end{array}$ & $\begin{array}{l}\text { Washington District, Jackson County, WV, } \\
\text { Kentuck, WV 7.5' quadrangle }\end{array}$ & $47-035-01366$ & 38.731 & -81.569 & 17,675 & |4/21/19975 (Run 1, FDC-GR-N), 8/26/1975 (Run 2, FDC-GR-N) & $0.17,700$ \\
\hline & A_Gainer_Lee & $\begin{array}{l}\begin{array}{l}\text { Exxon Corporation No.1 } \\
\text { Gainer-Lee }\end{array} \\
\end{array}$ & $\begin{array}{l}\text { Center District, Calhoun County, WV, } \\
\text { Grantsville, WV } 7.5 \text { quadrangle }\end{array}$ & $47-013-02503$ & 38.875 & -81.098 & 20,22 & 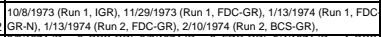 & $0-20,250$ \\
\hline & A_Gainer_Lee_B & $\begin{array}{l}\text { Exxon Corporation No.1 } \\
\text { Gainer-Lee }\end{array}$ & $\begin{array}{l}\text { Center District, Calhoun County, wV, } \\
\text { Grantsville, WV } 7.5^{\prime} \text { quadrangle }\end{array}$ & 47-013-02503 & 38.875 & -81.098 & 20,22 & 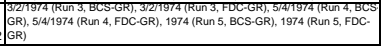 & $11,000-20,211$ \\
\hline & A_W_VA_Med_Security_Prison_A & $\begin{array}{l}\text { Hope Natural Gas No.10228 } \\
\text { WV Board of Control }\end{array}$ & $\begin{array}{l}\text { Huttonsville District, Randoloph County, WV, } \\
\text { Mill Creek, WV 7.5' quadrangle }\end{array}$ & 47-083-00103 & 38.707 & -79.969 & 13,121 & |5/8/1959 (Run 1, L-GR), 5/31/1959 (Run 2, GR) & $0.6,600$ \\
\hline 15 & A_W_VA_Med_Security_Prison_B & $\begin{array}{l}\text { Hope Natural Gas No.10228 } \\
\text { WV Board of Control }\end{array}$ & $\begin{array}{l}\text { Huttonsville District, Randolph County, WV, } \\
\text { Mill Creek, WV 7.5 quadrangle }\end{array}$ & 47-083-00103 & 38.707 & -79.969 & 13,12 & 11/17/1959 (Run 3, GR), 2/22/1960 (Run 2, L-GR-N) & 100- \\
\hline & A Sponangle & $\begin{array}{l}\text { United Fuel Gas Co. 8800-T } \\
\text { No.1 Ray Sponaugle }\end{array}$ & $\begin{array}{l}\text { Circleville District, Pendleton County, WV, } \\
\text { Snowy Mtn, WV } 7.5^{\circ} \text { quadrangle }\end{array}$ & 47-071-00006 & 38.548 & .79 .513 & 13,000 & $715 / 1960$ (Run 1, L-G, R-N) & $0.13,000$ \\
\hline
\end{tabular}


Scale, units, and depths for Gamma Ray logging runs:

\begin{tabular}{|c|c|c|c|c|}
\hline Well No. & Scale and Units & Depths of Selected Logged Intervals & Casing Shoe Location(s) & Notes \\
\hline 1 & $\begin{array}{l}0-200 \text { API units } \\
200-400 \text { backup scale }\end{array}$ & $\begin{array}{l}\text { About } 74 \text { feet below Kelly Busing (KB) } \\
\text { to Total Depth (TD) }\end{array}$ & & \\
\hline 2 & $\begin{array}{l}0-200 \text { API units } \\
200-400 \text { backup scale }\end{array}$ & 100 feet below KB to TD & & \\
\hline 3 & $0-200 \mathrm{API}$ units & Ground level (GL) to TD & & \\
\hline 4 & \begin{tabular}{|l}
$0-200$ API units \\
$200-400$ backup scale
\end{tabular} & 100 feet below KB to TD & & \\
\hline 5 & $\begin{array}{l}0-10 \text { (no units, probably } \\
\text { in micrograms of Radium } \\
\text { equivalent per metric ton } \\
\text { ( } \mu \text { gm Ra-eq/ton) } \\
10-20 \text { backup scale }\end{array}$ & About 9 feet below KB to TD & 1,245 feet below KB & \\
\hline 6 & $\begin{array}{l}0-200 \text { API units } \\
200-400 \text { backup scale }\end{array}$ & About 12 feet below KB to TD & 75 feet below KB & \\
\hline 7 & $\begin{array}{l}0-200 \text { API units } \\
200-400 \text { backup scale }\end{array}$ & 100 feet below KB to TD & 810 feet below KB & \\
\hline 8 & $\begin{array}{l}0-200 \text { API units } \\
200-400 \text { backup scale }\end{array}$ & 100 feet below KB to TD & 520 feet below KB & \\
\hline 9 & $\begin{array}{l}\text { 0-10 ( } \mu \text { gm Ra-eq/ton?) } \\
10-20 \text { backup scale }\end{array}$ & 100 feet below KB to 4,790 feet & 1,395 feet below KB & \\
\hline & $\begin{array}{l}0-8(\mu \text { gm } \mathrm{Ra}-\mathrm{eq} / \mathrm{ton} ?) \\
\text { 8-16 backup scale }\end{array}$ & 4,790 feet to TD & 4,790 feet below KB & \\
\hline 10 & $\begin{array}{l}\text { 0-200 API units } \\
\text { 200-400 backup scale }\end{array}$ & About 13 feet below KB to TD & $\begin{array}{c}116 \text { feet below KB } \\
2,006 \text { feet below KB } \\
6,891 \text { feet below KB }\end{array}$ & \\
\hline \multirow[t]{2}{*}{11} & $0-10 \mu \mathrm{gm}$ Ra-eq/ton & About 65 feet below KB to 12,330 feet & & \\
\hline & & 12,330 feet to TD & & Spontaneous Potential log (milivolts) not shown \\
\hline \multirow[t]{3}{*}{12} & \multirow{3}{*}{$\begin{array}{l}0-200 \text { API units } \\
200-400 \text { backup scale } \\
\text { (no scale change noted } \\
\text { between logging runs) }\end{array}$} & logging run 2: 2,390 to 8,710 feet & & \\
\hline & & logging run 3: 8,730 to 12,810 feet & & \\
\hline & & logging run 4: 12,810 feet to TD & & \\
\hline 13 & $\begin{array}{l}0-150 \text { API units } 150- \\
300 \text { backup scale }\end{array}$ & About 28 feet below KB to TD & & \\
\hline 14 & \begin{tabular}{|l|}
$0-120$ API units \\
$0-250$ API units \\
$0-200$ API units \\
$0-250$ API units \\
$0-150$ API units \\
\end{tabular} & $\begin{array}{l}\text { About } 27 \text { feet below KB to } 2,643 \text { feet } \\
2,670 \text { to } 8,190 \text { feet } \\
8,190 \text { to } 10,850 \text { feet } \\
10,850 \text { to } 11,020 \text { feet } \\
11,020 \text { feet to TD }\end{array}$ & & \\
\hline 15 & \begin{tabular}{|l|}
$0-15$ ( $\mu \mathrm{gm}$ Ra-eq/ton?) \\
$0-16$ ( $\mu \mathrm{gm}$ Ra-eq/ton?) \\
\end{tabular} & $\begin{array}{l}\text { About } 14 \text { feet below KB to } 6,300 \text { feet } \\
6,300 \text { feet to TD }\end{array}$ & & \\
\hline 16 & 0-12.5 ( $\mu \mathrm{gm}$ Ra-eq/ton?) & 100 feet below KB to TD & & \\
\hline
\end{tabular}




\begin{tabular}{|c|c|c|c|c|c|c|c|c|}
\hline Well \# & File Name & Well Name & Location & API No. & Latitude & Longitude & TD (ft) & LAS Interval (ft) \\
\hline & Haff_GRNeutron.las & East Ohio Gas Company No. 1-2171 Haff, V \& I & $\begin{array}{l}\text { TownsendTownship, Sandusky Co., OH } \\
\text { Clyde, OH 7.5' Quadrangle }\end{array}$ & 34-143-20077 & 41.371 & -82.907 & 3,123 & $0-3,200$ \\
\hline & Wheeler_GR.las & Pure Oil Company No. 1 Wheeler, I.M. & $\begin{array}{l}\text { GreenfieldTownship, Huron Co., OH } \\
\text { Willard, OH 7.5' Quadrangle }\end{array}$ & $34-077-20025$ & 41.106 & -82.704 & 3,865 & $100-3,900$ \\
\hline & Empire_Reeves.las & Empire Reeves Steel No. D-1 Empire Reeves Steel Division & $\begin{array}{l}\text { MadisonTownship, Richland Co., OH } \\
\text { Mansfield North, OH 7.5' Quadrangle }\end{array}$ & $34-139-20448$ & 40.779 & -82.519 & 5,085 & $0-5,100$ \\
\hline 4 & Drake.las & Great Lakes Gas Corporation No. 1 Drake, Alonzo, Jr. & $\begin{array}{l}\text { WayneTownship, Wayne Co., OH } \\
\text { Wooster, OH 7.5' Quadrangle } \\
\end{array}$ & $34-169-21419$ & 40.860 & -81.906 & 6,897 & $100-6,870$ \\
\hline & Troyer_first_part.las & Parker \& Chapman No. 1 Dan E Troyer & $\begin{array}{l}\text { SaltcreekTownship, Holmes Co., OH } \\
\text { Fredericksburg, OH 7.5' Quadrangle }\end{array}$ & $34-075-21283$ & 40.657 & -81.772 & 7,369 & $100-7,0$ \\
\hline & No_1_Troyer_last_300.las & Parker \& Chapman No. 1 Dan E Troyer & $\begin{array}{l}\text { SaltcreekTownship, Holmes Co., OH } \\
\text { Fredericksburg, OH 7.5' Quadrangle }\end{array}$ & $34-075-21283$ & 40.657 & -81.772 & 7,369 & $7,000-7,350$ \\
\hline 6] & HuebnerGR.las & Stocker \& Sitler, Inc. No. 2 (1-2669) Huebner & $\begin{array}{l}\text { RushTownship, Tuscarawas Co., OH } \\
\text { Gnadenhutton, OH 7.5' Quadrangle }\end{array}$ & $34-157-21030$ & 40.304 & -81.425 & 8,227 & $100-8,2$ \\
\hline & Zechman_A.las & Red Hill Development No. 1 Zechman, Thomas & $\begin{array}{l}\text { MoorefieldTownship, Harrison Co., OH } \\
\text { Piedmont, OH 7.5' Quadrangle }\end{array}$ & 34-067-20737 & 40.195 & -81.197 & 10,625 & $6,395-1$ \\
\hline & Zechman_B.las & Red Hill Development No. 1 Zechman, Thomas & $\begin{array}{l}\text { MoorefieldTownship, Harrison Co., OH } \\
\text { Piedmont, OH 7.5' Quadrangle }\end{array}$ & 34-067-20737 & 40.195 & -81.197 & 10,625 & $800-6,5$ \\
\hline 3 & Birney_A.LAS & McCormick Sanford E No. 1 Birney, Roy & $\begin{array}{l}\text { GreenTownship, Harrison Co., OH } \\
\text { Cadiz, OH 7.5' Quadrangle }\end{array}$ & $34-067-20103$ & 40.262 & -80.966 & 10,181 & $72-10,204$ \\
\hline & Birney_B.las & McCormick Sanford E No. 1 Birney, Roy & $\begin{array}{l}\text { GreenTownship, Harrison Co., OH } \\
\text { Cadiz, OH 7.5' Quadrangle }\end{array}$ & $34-067-20103$ & 40.262 & -80.966 & 10,181 & $8,900-10,050$ \\
\hline & Burley1.las & Sanford E McCormick No. 1 John Burley & $\begin{array}{l}\text { Liberty District, Marshall Co., WV } \\
\text { Cameron, WV 7.5' Quadrangle } \\
\end{array}$ & $47-051-00539$ & 39.762 & -80.530 & 16,512 & $50-10,500$ \\
\hline & Burley2.las & Sanford E McCormick No. 1 John Burley & $\begin{array}{l}\text { Liberty District, Marshall Co., WV } \\
\text { Cameron, WV 7.5' Quadrangle }\end{array}$ & $47-051-00539$ & 39.762 & -80.530 & 16,512 & $10,500-14,500$ \\
\hline 9 & Burley3.las & Sanford E McCormick No. 1 John Burley & $\begin{array}{l}\text { Liberty District, Marshall Co., WV } \\
\text { Cameron, WV 7.5' Quadrangle }\end{array}$ & $47-051-00539$ & 39.762 & -80.530 & 16,512 & $14,500-16,500$ \\
\hline 10 & Finch_A.las & Phillips Petroleum Co. No. A-1 (A-1251) R.R. Finch & $\begin{array}{l}\text { Winfield District, Marion Co., WV } \\
\text { Fairmont East, WV 7.5' Quadrangle }\end{array}$ & $47-049-00244$ & 39.432 & -80.012 & 17,111 & $0-10,500$ \\
\hline 10 & Finch_B.las & Phillips Petroleum Co. No. A-1 (A-1251) R.R. Finch & \begin{tabular}{|l|} 
Winfield District, Marion Co., WV \\
Fairmont East, WV 7.5' Quadrangle \\
\end{tabular} & $47-049-00244$ & 39.432 & -80.012 & 17,111 & $10,000-12,100$ \\
\hline 10 & Finch_C.las & Phillips Petroleum Co. No. A-1 (A-1251) R.R. Finch & $\begin{array}{l}\text { Winfield District, Marion Co., WV } \\
\text { Fairmont East, WV 7.5' Quadrangle }\end{array}$ & $47-049-00244$ & 39.432 & -80.012 & 17,111 & $12,100-14,900$ \\
\hline 10 & Finch_D.las & Phillips Petroleum Co. No. A-1 (A-1251) R.R. Finch & $\begin{array}{l}\text { Winfield District, Marion Co., WV } \\
\text { Fairmont East, WV 7.5' Quadrangle }\end{array}$ & $47-049-00244$ & 39.432 & -80.012 & 17,111 & $14,800-16,000$ \\
\hline 10 & Finch_E.las & Phillips Petroleum Co. No. A-1 (A-1251) R.R. Finch & $\begin{array}{l}\text { Winfield District, Marion Co., WV } \\
\text { Fairmont East, WV 7.5' Quadrangle }\end{array}$ & $47-049-00244$ & 39.432 & -80.012 & 17,111 & $15,900-17,000$ \\
\hline 10 & Finch_F.las & Phillips Petroleum Co. No. A-1 (A-1251) R.R. Finch & $\begin{array}{l}\text { Winfield District, Marion Co., WV } \\
\text { Fairmont East, WV 7.5' Quadrangle }\end{array}$ & $47-049-00244$ & 39.432 & -80.012 & 17,111 & $16,900-17,100$ \\
\hline 11 & Q-1_A.las & $\begin{array}{l}\text { Columbia Fuel Corp. No. USA Q-1, GW-1466 Monogahela National } \\
\text { Forest }\end{array}$ & $\begin{array}{l}\text { Union District, Preston Co., WV } \\
\text { Lead Mine, WV 7.5' Quadrangle }\end{array}$ & $47-077-00119$ & 39.238 & -79.573 & 9,910 & $0-7,550$ \\
\hline 11 & Q-1_B.las & $\begin{array}{l}\text { Columbia Fuel Corp. No. USA Q-1, GW-1466 Monogahela National } \\
\text { Forest }\end{array}$ & $\begin{array}{l}\text { Union District, Preston Co., WV } \\
\text { Lead Mine, WV 7.5' Quadrangle }\end{array}$ & $47-077-00119$ & 39.238 & -79.573 & 9,910 & $7,700-10,000$ \\
\hline 11 & Q-1_GR_D_final.las & $\begin{array}{l}\text { Columbia Fuel Corp. No. USA Q-1, GW-1466 Monogahela National } \\
\text { Forest }\end{array}$ & $\begin{array}{l}\text { Union District, Preston Co., WV } \\
\text { Lead Mine, WV 7.5' Quadrangle }\end{array}$ & $47-077-00119$ & 39.238 & -79.573 & 9,910 & $0-9,910$ \\
\hline 12 & greenland_GR.las & $\begin{array}{l}\text { Shell Oil Co. (Consolidated Gas Supply Corp.) No. } 1 \text { Greenland Lodge, } \\
\text { Inc. }\end{array}$ & $\begin{array}{l}\text { Union District, Grant Co., WV } \\
\text { Greenland Gap, WV 7.5' Quadrangle }\end{array}$ & $47-023-00002$ & 39.195 & -79.142 & 16,075 & $50-13,000$ \\
\hline 13 & Bean A.las & Exxon Corporation No. 1 Charles H. Bean, et al. & $\begin{array}{l}\text { Moorefield District, Hardy Co., WV } \\
\text { Old Fields, WV 7.5' Quadrangle }\end{array}$ & 47-031-00021 & 39.138 & -78.990 & 16,075 & $100-5,300$ \\
\hline 13 & Bean_B.las & Exxon Corporation No. 1 Charles H. Bean, et al. & $\begin{array}{l}\text { Moorefield District, Hardy Co., WV } \\
\text { Old Fields, WV 7.5' Quadrangle }\end{array}$ & $47-031-00021$ & 39.138 & -78.990 & 16,075 & $5,300-10,450$ \\
\hline 13 & Bean_C.las & Exxon Corporation No. 1 Charles H. Bean, et al. & $\begin{array}{l}\text { Moorefield District, Hardy Co., WV } \\
\text { Old Fields, WV 7.5' Quadrangle }\end{array}$ & 47-031-00021 & 39.138 & -78.990 & 16,075 & $10,450-16,050$ \\
\hline
\end{tabular}


Scale, units, and depths for Gamma Ray logging runs. Abbreviations: API, American Petroleum Institute; KB, Kelly bushing; TD, total depth

\begin{tabular}{|c|c|c|c|}
\hline Well No. & Scale and Units & Depths of Selected Logged Intervals & Casing Shoe Location(s) \\
\hline 1 & $\begin{array}{l}\text { 0-12 micro Roentgens / } \\
\text { hour }(\mu \mathrm{R} / \mathrm{hr})\end{array}$ & $\begin{array}{l}10 \text { feet below Kelly Bushing (KB) } \\
\text { to Total depth (TD) }\end{array}$ & \\
\hline 2 & $\begin{array}{l}\text { 0-200 API units } \\
\text { 200-400 backup scale }\end{array}$ & 100 feet below KB to TD & 473 feet below KB \\
\hline 3 & $0-200$ API units & About 7 feet below KB to TD & \\
\hline 4 & $\begin{array}{l}\text { 0-200 API units } \\
\text { 200-400 backup scale }\end{array}$ & 100 feet below KB to TD & 3,615 feet below KB \\
\hline 5 & $\begin{array}{l}\text { 0-200 API units } \\
200-400 \text { backup scale }\end{array}$ & 100 feet below KB to TD & 1,220 feet below KB \\
\hline 6 & $\begin{array}{l}\text { 0-200 API units } \\
\text { 200-400 backup scale }\end{array}$ & 100 feet below KB to TD & 1,420 feet below KB \\
\hline 7 & $0-200$ API units & 828 feet below KB to TD & 6,416 feet below KB \\
\hline 8 & $\begin{array}{l}\text { 0-200 API units } \\
\text { 200-400 backup scale }\end{array}$ & 60 feet below KB to TD & 1,590 feet below KB \\
\hline 9 & $\begin{array}{l}\text { 0-250 API units } \\
0-250 \mathrm{API} \text { units } \\
\text { 0-250 API units } \\
\text { Units not listed (probably } \\
\text { 0-250 API units) }\end{array}$ & $\begin{array}{l}80 \text { feet below } K B \text { to } 3,870 \text { feet } \\
3,870 \text { feet below KB to } 10,505 \text { feet } \\
10,505 \text { feet below KB to } 14,475 \text { feet } \\
10,505 \text { feet below KB to TD }\end{array}$ & $\begin{array}{l}100 \text { feet below } K B \\
3,903 \text { feet below KB } \\
10,505 \text { feet below KB }\end{array}$ \\
\hline 10 & $\begin{array}{l}0-150 \text { API units } \\
0-200 \text { API units } \\
0-150 \text { API units } \\
0-200 \text { API units } \\
0-150 \text { API units }\end{array}$ & $\begin{array}{l}\text { KB to } 990 \text { feet } \\
990 \text { feet below } K B \text { to } 7,455 \text { feet } \\
7,455 \text { feet below } K B \text { to } 7,502 \text { feet } \\
7,502 \text { feet below } K B \text { to } 12,075 \text { feet } \\
12,075 \text { feet below KB to TD }\end{array}$ & 993 feet below KB \\
\hline 11 & $\begin{array}{l}0-150 \text { API units } \\
150-300 \text { backup scale } \\
0-200 \text { API units }\end{array}$ & $\begin{array}{l}\text { KB to } 7,710 \text { feet } \\
7,710 \text { feet below KB to TD }\end{array}$ & $\begin{array}{l}1,215 \text { feet below KB } \\
4,925 \text { feet below } K B\end{array}$ \\
\hline 12 & $\begin{array}{l}0-200 \text { API units } \\
0-150 \text { API units } \\
0-200 \text { API units }\end{array}$ & $\begin{array}{l}50 \text { feet below KB to } 480 \text { feet } \\
480 \text { feet below KB to } 10,021 \text { feet } \\
10,021 \text { feet below KB to TD }\end{array}$ & $\begin{array}{l}336 \text { feet below } K B \\
5,255 \text { feet below } K B\end{array}$ \\
\hline 13 & $\begin{array}{l}0-200 \mathrm{API} \text { units } \\
\text { Units not listed (probably } \\
0-250 \mathrm{API} \text { units) } \\
0-200 \mathrm{API} \text { units }\end{array}$ & $\begin{array}{l}100 \text { feet below KB to } 1,490 \text { feet } \\
1,490 \text { feet below KB to } 5,920 \text { feet } \\
5,920 \text { feet below KB to TD }\end{array}$ & 6.000 feet below KB \\
\hline
\end{tabular}

